\title{
The utility of cardiac magnetic resonance imaging in Kounis syndrome
}

\author{
Aylin Okur ${ }^{1}$, Mecit Kantarci², Leyla Karaca², Hayri Ogul², Ayhan Aköz³, Yesim Kızrak², Sahin Aslan³, \\ Berhan Pirimoglu², Enbiya Aksakal ${ }^{4}$, Mucahit Emet $^{3}$ \\ ${ }^{1}$ Department of Radiology, School of Medicine, Bozok University, Yozgat, Turkey \\ 2Department of Radiology, School of Medicine, Ataturk University, Erzurum, Turkey \\ ${ }^{3}$ Department of Emergency Medicine, School of Medicine, Ataturk University, Erzurum, Turkey \\ ${ }^{4}$ Department of Cardiology, School of Medicine, Ataturk University, Erzurum, Turkey
}

Postep Kardiol Inter 2015; 11, 3 (41): 218-223

DOI: $10.5114 /$ pwki.2015.54017

\begin{abstract}
A b stract
Introduction: Current diagnostic measurements used to assess myocardial involvement in Kounis syndrome, such as electrocardiography (ECG), cardiac enzymes, and troponin levels, are relatively insensitive to small but potentially significant functional change. According to our review of the literature, there has been no study using magnetic resonance imaging (MRI) on Kounis syndrome except for one case report.

Aim: To identify the findings of dynamic contrast-enhanced magnetic resonance imaging (CE-MRI) in patients with Kounis syndrome (KS) type 1.

Material and methods: We studied 26 patients ( $35 \pm 11.5$ years, $53.8 \%$ male) with known or suspected KS type 1 . The patients underwent precontrast, first-pass, and delayed enhancement cardiac MRI (DE-MRI). Contrast enhancement patterns, edema, hypokinesia, and localization for myocardial lesions were evaluated in all KS type 1 patients.

Results: Contrast-enhanced magnetic resonance imaging demonstrated an early-phase subendocardial contrast defect, and T2-weighted images showed high-signal intensity consistent with edema in lesion areas. None of the lesion areas was found upon contrast enhancement on DE-MRI. The area of early-phase subendocardial contrast defect was reported as follows: the interventricular septum in 14 (53.8\%) patients, the left ventricular lateral wall in 8 (30.7\%), and the left ventricular apex in 4 (15.4\%).

Conclusions: Dynamic cardiac MR imaging is a reliable tool for assessing cardiac involvement in Kounis syndrome. Delayed contrast-enhanced images show normal washout in the subendocardial lesion area in patients with Kounis syndrome type 1.
\end{abstract}

Key words: Kounis syndrome, hypersensitivity, cardiac magnetic resonance imaging.

\section{Introduction}

Kounis syndrome (KS) is the concurrence of acute coronary vasospasm with conditions associated with mast cell degranulation and the release of inflammatory mediators during an allergic reaction. Allergic or hypersensitivity mediators, such as various drugs, conditions, and environmental exposures, trigger coronary vasoconstriction and induce plaque rupture [1]. Kounis syndrome has been increasingly reported in clinical practice. Its true frequency is not precisely known [2].

Kounis syndrome is divided into three subgroups: type I occurs in patients with normal coronary arteries in whom the acute release of inflammatory mediators induces coronary artery spasm. This type represents a manifestation of endothelial dysfunction or microvascular angina. Type 2 patients have preexisting plaques and coronary spasm occurs adjacent to dormant atheromatous plaques in which allergic stimulation results in plaque erosion or rupture leading to myocardial infarction [3]. Recently, type $3 \mathrm{KS}$ has been reported in patients with coincidence of hypersensitivity reaction following implantation of drug-eluting stents and stent thrombosis [4].

Generally, ST elevations on electrocardiogram (ECG), increased cardiac enzymes and coronary angiography findings were reported in previously published cases of Kounis syndrome diagnosis [2, 5]. These techniques have been proven to be clinically useful, but each has limitations that may reduce its diagnostic accuracy. For

\section{Corresponding author:}

Mecit Kantarci MD, Department of Radiology, School of Medicine, Ataturk University, 200 Evler Mah. 14. Sok No 5, Dadaskent, Erzurum, Turkey, phone: +90 442 2361212-1521, fax: +90 (442) 2361301, e-mail: akkanrad@hotmail.com

Received: 2.05.2014, accepted: 12.06.2014. 
example, none of these procedures can assess the viability of the ventricular wall. In cardiac diseases, assessing the viability of affected myocardial tissue is an important prognostic predictor of recovery following revascularization [6, 7]. Moreover, previous studies have also shown normal ECG findings, cardiac enzymes, and coronary angiography in Kounis syndrome type 1 cases $[2,8]$. Novel complementary techniques are needed to support clinical and laboratory findings in patients with suspected Kounis syndrome in the assessment of myocardial viability.

According to our review of literature, there has been no study on Kounis syndrome using magnetic resonance imaging (MRI) except for one case report [9]. Recently, MRI has become an important diagnostic tool in cardiac imaging. Magnetic resonance imaging not only evaluates myocardial perfusion but also demonstrates the morphology and function of myocardial tissue [10, 11]. Magnetic resonance imaging with a gadolinium-enhanced contrast agent offers high spatial resolution and can differentiate between non-ischemic and ischemic heart disease [12].

\section{Aim}

Inspired by this information, we evaluated patients who attended the Emergency or Cardiology Department with a clinical diagnosis of Kounis syndrome by cardiac MRI (CMRI) and aimed to identify CMRI findings in patients with known or suspected Kounis syndrome type 1 with an emphasis on the additive clinical information.

\section{Material and methods}

Between March 2012 and August 2013, 33 consecutive patients (18 men, 15 women) whose age ranged from 18 to 57 years (mean: $36 \pm 11.3$ years) underwent CMRI for chest pain, which occurred simultaneously with the symptoms of allergies (i.e. hives, erythroderma, uvula edema). All patients underwent coronary computed tomographic angiography (CTA) and CMRI (with and without contrast enhancement) within $24 \mathrm{~h}$ after clinical evaluation.

Patients who had at least one of the disorders of the ECG or cardiac enzymes (creatine phosphokinase, creatine kinase- $M B$, and troponin I), and normal coronary CTA, in addition to chest pain and allergic clinical symptoms, were included in this study. Seven patients were excluded due to a history of myocardial infarction/coronary artery disease $(n=2)$, claustrophobia $(n=2)$, age under 18 or over 60 years $(n=2)$, and poor image quality $(n=1)$. The final study cohort consisted of 26 patients (14 men, 12 women; mean age $35 \pm 11.5$ years).

This prospective study was approved by the institutional ethics committee, and written informed consent was obtained from all patients before enrollment.

\section{Computed tomographic angiography imaging}

All patients were scanned with 256-slice multidetector computed tomography (MDCT) (Siemens Medical Systems, Somatom, Definition Flash, Forchheim, Germany). Scan parameters were 120 kV, 340 mAs, 420 ms rotation time with a slice thickness of $1 \mathrm{~mm}$ and increments of $0.5 \mathrm{~mm}$, using a detector collimation of $16 \times 0.75 \mathrm{~mm}$ (pitch: 0.2). The mean heart rate was 65 beats per minute during acquisition. Seventy milliliters of non-ionic, iodinated, low-osmolar contrast medium (Ultravist 370 mg/ml, Bayer Schering Pharma, Berlin, Germany) was injected through the antecubital vein at a rate of $5 \mathrm{ml} / \mathrm{s}$. Subsequently, a bolus of $40 \mathrm{ml}$ saline solution was administered at a rate of $2.5 \mathrm{ml} / \mathrm{s}$ to avoid possible contrast artifacts at the right atrial entrance. An automatic bolus tracking method was used to optimize visualization. In addition to traditional axial images, other available techniques including multiplanar reconstruction (MPR) and three-dimensional volume rendering (3D VR) images were performed.

\section{Magnetic resonance imaging}

All patients were examined with a 1.5-T MR scanner (Siemens AG, Magnetom Avanto, 2008, Germany) with an 8-channel cardiac coil placed around the patient's chest. Localization was performed using breath-hold real-time and steady-state free-precession (SSFP) images of true anatomic axes of the heart. All of the patients underwent precontrast, first-pass, delayed MR images, and T2 weighted (T2w) fast spin echo sequences were performed in axial and coronal planes for localization using electrocardiographic-gated (ECG) cardiac MR.

After two rapid surveys to determine the exact heart axis, 8-10 short-axis planes (apical, midventricular, and basal) as well as four- and two-chamber views were acquired. The imaging parameters are as follows: TR/TE, 770/35; slice thickness, 3-5 mm; matrix, $192 \times 256$; contiguous sections, 10-14; slice gap, $0 \mathrm{~mm}$; temporal resolution $40 \mathrm{~ms}$; and number of phases, 20.

Imaging parameters for $\mathrm{T} 2 \mathrm{~W}$ images were: TR/TE, $61 / 2 \mathrm{~ms}$; inversion time, $170 \mathrm{~ms}$; flip angle, $180^{\circ}$; matrix, $256 \times 256$; slice thickness, $6-8 \mathrm{~mm}$.

In our first-pass MR imaging protocol, four images ( $3 \times$ short axis (apical, midventricular, and basal) + 4-chamber view) of a single-shot turbo Fast low angle shot (FLASH) sequence with saturation recovery preparation (TR/TE, 298/1.66 ms; inversion time, $200 \mathrm{~ms}$; flip angle, $12^{\circ}$; temporal resolution, $1.5 \mathrm{~s}$; matrix, $96 \times 128$; slice thickness, $6-8 \mathrm{~mm}$ ) displaying the optimal view of the related pathology were chosen from the cine and black blood studies.

Delayed enhancement magnetic resonance imaging was performed $10 \mathrm{~min}$ after $0.2 \mathrm{mmol} / \mathrm{kg}$ injection of gadopentetate dimeglumine (Gd-DTPA, Magnevist, Bayer Healthcare, Germany). The DE-MRI protocols in- 
clude the following: TR/TE, 298/1.66 ms; inversion time, $200 \mathrm{~ms}$; flip angle, $12^{\circ}$; temporal resolution, $1.5 \mathrm{~s}$; matrix, $96 \times 128$; slice thickness, 6-8 mm. Reduction of breathing artifacts was performed by breath holding in end-expira-

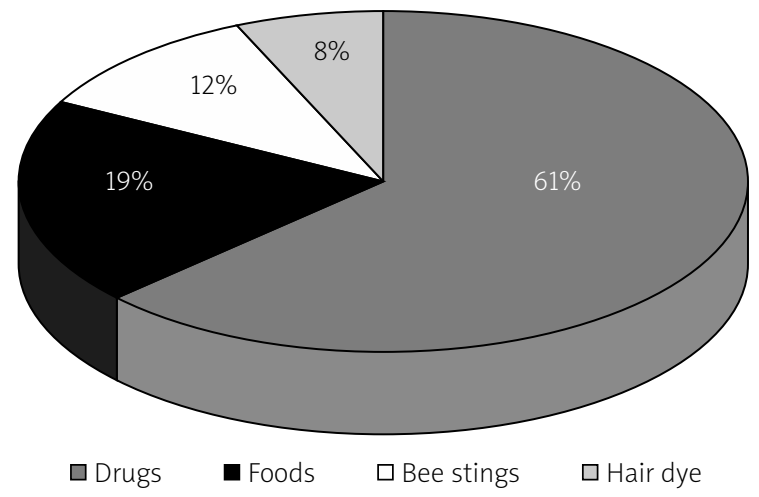

Figure 1. Distribution of allergic agents in Kounis syndrome type 1

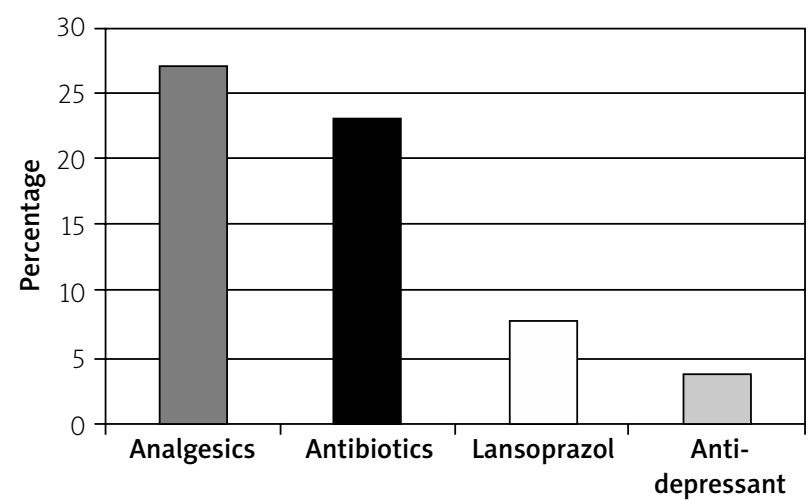

Figure 2. Prevalence of drugs among allergic agents

Table I. Demographics and cardiac MRI characteristics of patients with Kounis syndrome

\begin{tabular}{lc}
\hline Characteristics & Patients $(n=26)$ \\
\hline \begin{tabular}{l} 
Demographics: \\
\hline Age [years]
\end{tabular} & $35 \pm 11.5$ \\
\hline Male sex, $n(\%)$ & $18(53.8)$ \\
\hline MRI finding: & $26(100)$ \\
\hline First-pass contrast defect (\%) & $26(100)$ \\
\hline Lack of late gadolinium enhancement (\%) & $26(100)$ \\
\hline T2 edema, $n$ (\%) & $12(46)$ \\
\hline Hypokinesia, $n$ (\%) & $14(53.8)$ \\
\hline Interventricular septum involvement, $n(\%)$ & $8(30.7)$ \\
\hline Left ventricle lateral wall involvement, $n(\%)$ & $4(15.4)$ \\
\hline Apex involvement, $n(\%)$ &
\end{tabular}

tion during scanning. ECG rhythm, blood pressure, and symptoms were continuously monitored.

\section{Image evaluation}

All MR and CT images were transferred to a workstation (Syngo Via Console, software version 2.0; Siemens AG Medical Solutions, Germany) for evaluation, and two experienced cardiovascular radiologists reviewed all images independently. Interpretation discrepancies were resolved by means of a consensus.

Quantification of LV volumes and ejection fraction (EF) was performed as previously described using Argus software (Version 2002B, Siemens Medical Solutions) [12] on cine MR images. The evaluation parameters were: (1) myocardial perfusion defect in the early phase (firstpass) of contrast agent; (2) characteristics of myocardial contrast enhancement in delayed MR images; (3) edema in T2-weighted images; (4) hypokinesia in cine MR images; (5) localization of defect (subendocardial, transmural, or subepicardial); and (6) calculation of EF.

In addition, the patency of coronary arteries was assessed on CTA images.

\section{Statistical analysis}

The definitive statistical analysis of this study was made using the SPSS version 20.0 for Windows (Chicago, Illinois) statistical software. The normality of the variables was analyzed using the Kolmogorov-Smirnov test. There were no normally distributed numerical variables. Non-normally distributed numerical variable (EF) values are shown as medians (minimum-maximum), and categorical variables are shown as percentages. We used the $\chi^{2}$ test to analyze the categorical variables. The one-sample $T$ test was also used to analyze the numerical variable (ejection fraction values).

\section{Results}

Fourteen (53.8\%) of 26 patients were men. The most common cause of the allergy was drugs (Figure 1), and this has been reported in 16 patients (analgesics in 7 , antibiotics in 6, lansoprazole in 2, antidepressant in 1). Other agents are shown in Figure 2 (food allergy in 5 patients, bee sting in 3 patients, and hair dye in 2 patients).

ST elevation in ECG in 9 patients, nonspecific ECG in 10 patients, tachycardia in 9 patients, bradycardia in 2 patients, atrial fibrillation in 1 patient, and normal ECG in 6 patients were found. All patients were kept under surveillance at the hospital for approximately $24 \mathrm{~h}$. All patients were discharged from hospital with full recovery. The diameters of all coronary arteries were in the reference range without prominent plaque.

Demographic characteristics and MRI findings are shown in Table I. The CE-MRI demonstrated earlyphase (first-pass) subendocardial contrast defect, and T2-weighted images showed high signal intensity consis- 

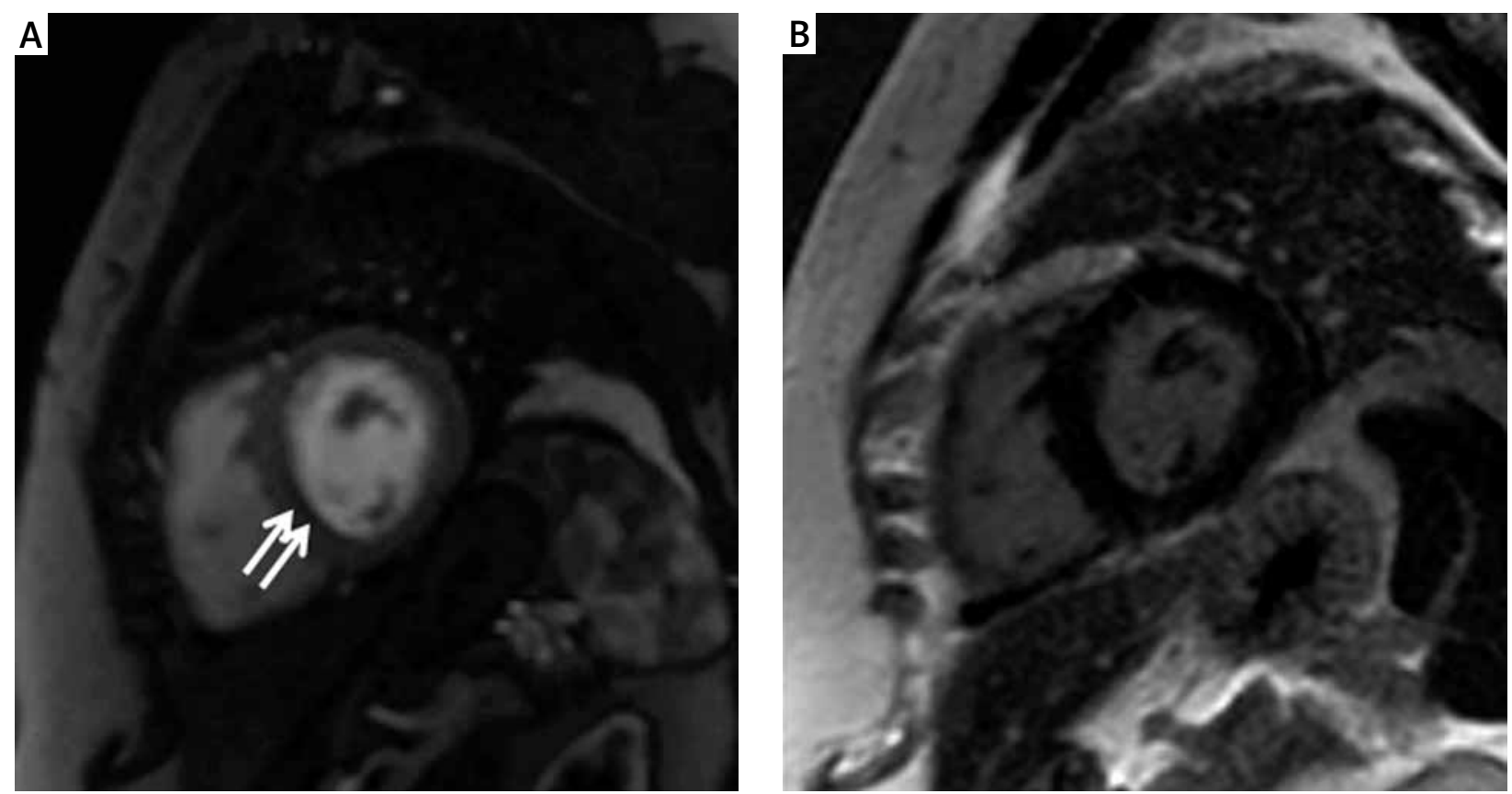

Figure 3. In interventricular septum. A - First-pass image shows hypointense subendocardial contrast defect (arrows). B - Normal washout of contrast seen on DE-MR image

tent with edema for all lesion areas. No transmural myocardial involvement was detected in any of the patients. Early-phase subendocardial contrast defect was reported as follows: the interventricular septum in 14 (53.8\%) patients (Figures $3 \mathrm{~A}, \mathrm{~B}$ ), the left ventricular lateral wall in $8(30.7 \%)$ patients, and the left ventricular apex in $4(15.4 \%)$ patients (Figures 4 A, B). None of the lesion areas were found upon contrast enhancement on DE-MRI. Moreover, hypokinesia was demonstrated in 12 (46\%) patients in the contrast defect area.

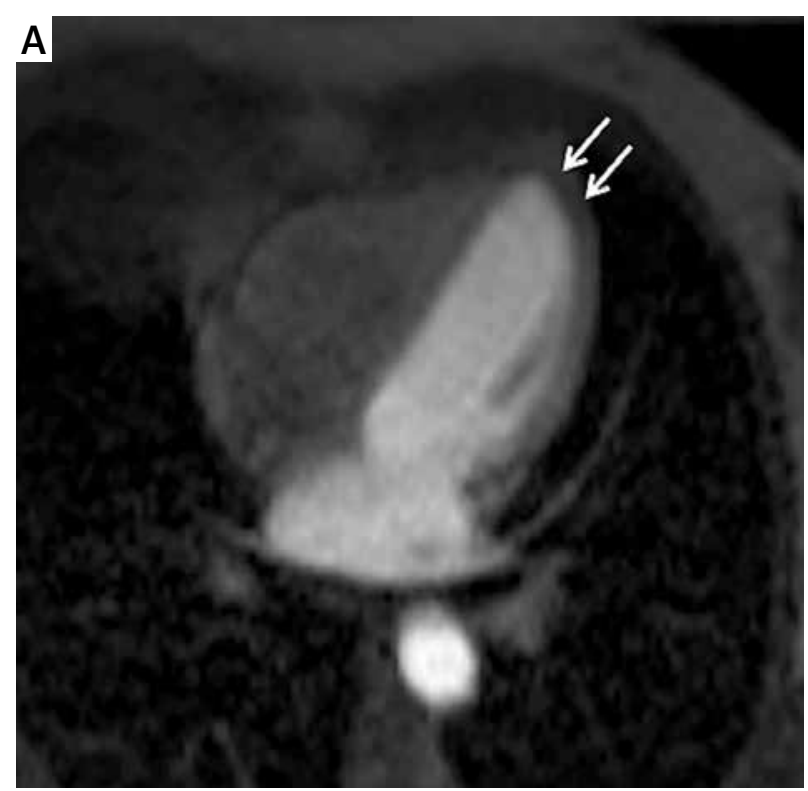

All patients revealed EF values within the normal range $(60-75 \%)$. Scanning times of the examinations were between 25 and 30 min. All patients underwent the complete examination without complications. None of the patients developed MI or death.

\section{Discussion}

To the best of our knowledge, the present study is the first original study describing CMRI findings in KS type 1 patients. This study has demonstrated that subendocar-

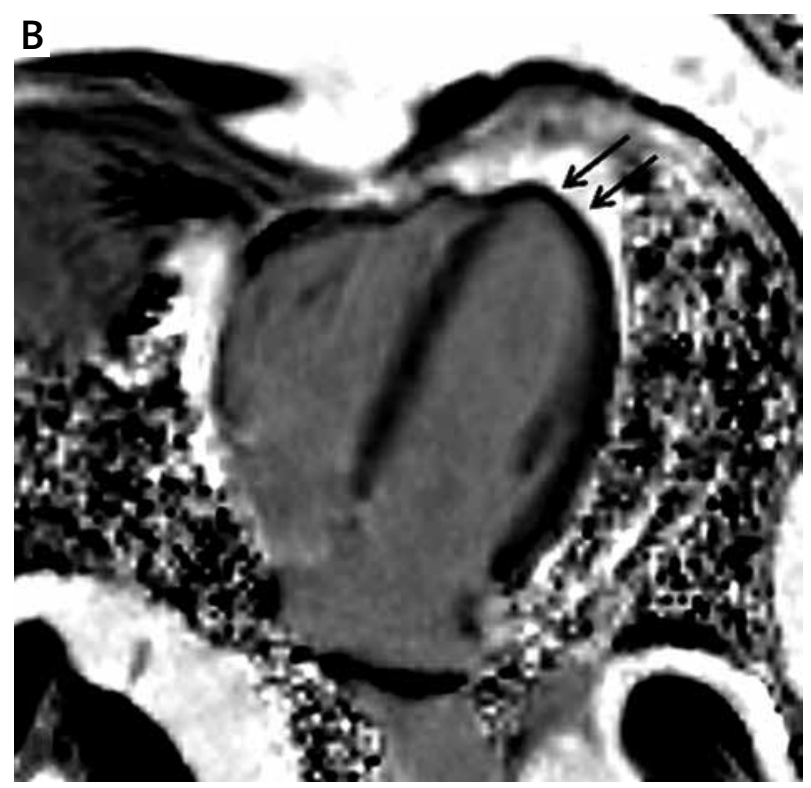

Figure 4. In left ventricular apex. A - First-pass image shows hypointense subendocardial contrast defect (white arrows). B - Normal washout of contrast seen on DE-MR image (black arrows) 
dial involvement, contrast defect on first-pass MR images, normal washout of contrast agent on delayed images, and sensitivity to the left ventricle are specific findings for patients with Kounis syndrome type 1 .

Current diagnostic measurements used to assess myocardial involvement in Kounis syndrome, such as ECG, cardiac enzymes, and troponin levels, are relatively insensitive to small but potentially significant functional change. In a study, two cases reported that cardiac biomarkers and electrocardiographic changes do not lead as they guide in KS [9]. Therefore, biopsy may also be necessary to characterize the underlying cause of myocardial disease, such as myocarditis and Kounis syndrome.

In these circumstances, cardiac imaging can help provide supportive diagnostic information. Echocardiography or single-photon emission computed tomography (SPECT) may miss myocardial involvement, particularly when it is small or subendocardial due to low spatial resolution [11]. Coronary CT angiography is highly valuable, but its value is limited for characterization of myocardial lesions. It needs a contrast agent and has radiation exposure [12]. The newer imaging modalities, such as MRI, offer an overall assessment of the myocardial tissue. Advantages of MRI are its non-invasiveness, non-ionizing, multiplanar capability, and excellent soft tissue contrast. Cardiac MRI also maintains high spatial and temporal resolution, and can therefore depict myocardial structure and function in great detail. Previous studies showed that CE-MRI added diagnostic information over clinical examination for patients with symptoms of acute coronary syndrome [13].

Hypersensitivity myocarditis and KS are two cardiac entities of allergic etiology affecting the myocardium and coronary arteries, respectively [2]. The myocardium and the coronary arteries can be particularly affected by hypersensitivity. Moreover, allergic reactions may also progress to myocardial infarction [14]. Therefore, differential diagnosis is very important [2]. Kounis syndrome is asynchronization of an acute coronary entity with mast cell degranulation via inflammatory mediators released during an allergic and anaphylactic reaction [15, 16]. These two entities can mimic each other and be clinically indistinguishable. The presence of eosinophils, atypical lymphocytes, and giant cells on myocardial biopsy suggests hypersensitivity myocarditis, whereas the biopsy is typically normal in KS [2]. However, this procedure is invasive and has not been widely available in daily clinical practice.

Recently, cardiac MRI has been used in a growing number of clinical applications for cardiac diseases. The assessment of the viability of affected myocardial tissue is very important for recovery following revascularization. It is possible to distinguish between viable and non-viable myocardial tissue using dynamic contrast-enhanced MRI [7]. Cardiac MRI may also depict ventricular volume, ejection fraction, ventricular mass, and infarct size. Fol- lowing the administration of contrast, the myocardium typically shows homogeneous enhancement, and appears washed out on delayed images on CMR images, whereas contrast enhancement is not detected in ischemic myocardial tissue on first-pass perfusion images. Delayed myocardial enhancement MRI has been developed primarily for description of myocardial scar tissue. The myocardial infarction or scar due to ischemic cardiomyopathies is identified by late gadolinium enhancement on cardiac magnetic resonance imaging [17]. The ischemic areas are known to be localized in the subendocardial area which is definitely conforming to one of the particular coronary territories. We did not detect any subendocardial contrast enhancement on delayed-phase images in myocardial tissue, unlike myocardial infarction.

Previous studies have reported that CMRI can differentiate acute myocarditis from myocardial infarction [18]. In acute myocarditis, the presence of delayed enhancement is patchy and does not conform to any particular coronary artery distribution. It usually appears in the subepicardial or mid-wall myocardial layers and never in the subendocardial area alone [19]. In our study, the myocardial lesions were localized in the subendocardial area on first pass MR images and definitely conformed to one of the particular coronary territories in all patients, unlike myocarditis.

Edema is a generic tissue response to acute insults and a nonspecific finding for myocardial disease [18]. T2-weighted imaging is the standard imaging technique used to detect acute myocardial edema. Myocardial edema can be detected by T2-weighted images as early as $30 \mathrm{~min}$ after the onset of ischemia, and is thought to represent reversible myocardial injury in the absence of late gadolinium enhancement $[20,21]$. In our study, T2-weighted images of edema were evident in the lesion area in all patients.

While coronary arteries are normal in type $1 \mathrm{KS}$, type 2 patients have preexisting plaques [5]. In the present study, there was no prominent plaque in the coronary arteries of any of the patients on CTA images.

In light of these findings, we evaluated lesion areas as hibernating myocardium tissue and identified the myocardial involvement of Kounis syndrome type 1 . Hibernating myocardial tissue is viable and can be restored toward normal by revascularization. It is always in the subendocardial area and conforms to a particular coronary territory, and contrast enhancement is unexpected in late-phase MR images [18] as in our study. Hibernating myocardial tissue has decreased myocardial contractile function (hypokinesia or dyskinesia) and reduced coronary blood flow owing to coronary arterial spasm. The reduced coronary blood flow causes the myocytes to enter into a low-energy state. This study demonstrated hypokinesia in $46 \%$ of the affected areas.

Previous studies performed on the basis of ECG showed that the right coronary artery spasm was more 
frequently determined than the left coronary artery spasm in Kounis syndrome [22]. In the present study, we found that the septum and the left ventricular lateral wall were susceptible for all patients with Kounis syndrome. We speculated that the explanation for this septal involvement would be the diameter of the perforating branches. Because the perforating branches which supply the septum have lesser calibration, they may be particularly sensitive to vasospasm. However, we did not explain the left ventricular lateral wall involvement by the same mechanism.

Our study has several limitations. Firstly, the study was not blinded. It would be difficult to disregard available information in patients presenting to the emergency department with a suspicion of acute coronary syndrome. Secondly, this study was a cross-sectional study with a small sample size. Thirdly, we did not provide histopathological confirmation of our findings. Finally, we could not evaluate the response to treatment as clinical because follow-up MRI was not performed.

\section{Conclusions}

Contrast-enhanced magnetic resonance imaging allows the assessment of the heart of patients with known or suspected KS type 1. Cardiac MRI may be performed in patients presenting with chest pain and allergy in the emergency department and could be a complementary procedure to clinical and laboratory findings for clarification in cases of diagnostic uncertainty, such as myocarditis or Kounis syndrome. We believe that our study may guide further research on KS and contribute to the diagnosis of Kounis syndrome type 1.

\section{Conflict of interest}

The authors declare no conflict of interest.

\section{References}

1. Kounis NG. Cardiovascular disease and allergy: angina pectoris, myocardial infarction. In: The experts speak: the role of nutrition in medicine. Hamilton K, Simpson K (eds.). Sacramento, Calif: IT Services 1997; 36.

2. Lopez PR, Peiris AN. Kounis syndrome. South Med J 2010; 103: 1148-55.

3. Biteker M. Current understanding of Kounis syndrome. Expert Rev Clin Immunol 2010; 6: 777-88.

4. Biteker M. A new classification of Kounis syndrome. Int J Cardiol 2010; 145: 553.

5. Park JM, Cho J, Chung SP, Kim MJ. Kounis syndrome captured by coronary angiography computed tomography. Am J Emerg Med 2010; 28: 640.e5-8.

6. Dilsizian V, Rocco TP, Freedman NMT, et al. Enhanced detection of ischemic but viable myocardium by the reinjection of thallium after stress-redistribution imaging. N Engl J Med 1990; 323: 141-6.
7. Fatih Karakas M, Bilen E, Kurt M, et al. The correlation between infarct size and the QRS axis change after thrombolytic therapy in ST elevation acute myocardial infarction. EAJM 2012; 44: 13-7.

8. Emet M, Kantarci M, Aksakal E, et al. Allergic angina can be determined by the early use of cardiac magnetic resonance imaging. Am J Emerg Med 2010; 28: 1061.e5-7.

9. Akoz A, Bayramoglu A, Uzkeser M, et al. Two questions for Kounis syndrome: can we use magnetic resonance imaging in the diagnosis and does ST elevation correlates with troponin levels? Am J Emerg Med 2012; 30: 2086.e5-7.

10. Gerber BL, Raman SV, Nayak K, et al. Myocardial first-pass perfusion cardiovascular magnetic resonance: history, theory, and current state of the art. J Cardiovasc Magn Reson 2008; 10: 18.

11. Kim HW, Farzaneh-Far A, Kim RJ. Cardiovascular magnetic resonance in patients with myocardial infarction: current and emerging applications. J Am Coll Cardiol 2009; 55: 1-16.

12. Schoenhagen P, Dewey M. CT Assessment of coronary artery disease: trends and clinical implications. JACC Cardiovasc Imaging 2013; 6: 1072-4.

13. Kwong RY, Schussheim AE, Rekhraj S, et al. Detecting acute coronary syndrome in the emergency department with cardiac magnetic resonance imaging. Circulation 2003; 107: 531-7.

14. Almpanis GC, Mazarakis A, Dimopoulos DA, et al. The conundrum of hypersensitivity cardiac disease: hypersensitivity myocarditis, acute hypersensitivity coronary syndrome (Kounis syndrome) or both? Int J Cardiol 2011; 148: 237-40.

15. Sinkiewicz W, Sobanski P, Bartuzi Z. Allergic myocardial infarction. Cardiol J 2008; 15: 220-5.

16. Kounis NG, Zavras GM. Histamine-induced coronary artery spasm: the concept of allergic angina. Br J Clin Pract 1991; 45: 121-8.

17. Reimer KA, Lowe JE, Rasmussen MM, Jennings RB. The wavefront phenomenon of ischemic cell death. 1. Myocardial infarct size vs duration of coronary occlusion in dogs. Circulation 1977; 56 : 786-94.

18. Vogel-Claussen J, Rochitte CE, Wu KC, et al. Delayed enhancement MR imaging: utility in myocardial assessment. Radiographics 2006; 26: 795-810.

19. Friedrich MG. Tissue characterization of acute myocardial infarction and myocarditis by cardiac magnetic resonance. JACC Cardiovasc Imaging 2008; 1: 652-62.

20. Aletras AH, Tilak GS, Natanzon A, et al. Retrospective determination of the area at risk for reperfused acute myocardial infarction with T2-weighted cardiac magnetic resonance imaging: histopathological and displacement encoding with stimulated echoes (DENSE) functional validations. Circulation 2006; 113: 1865-70.

21. Abdel-Aty H, Cocker M, Meek C, et al. Edema as a very early marker for acute myocardial ischemia: a cardiovascular magnetic resonance study. J Am Coll Cardiol 2009; 53: 1194-201.

22. Rico Cepeda P, Palencia Herrejón E, Rodríguez Aguirregabiria MM. [Kounis syndrome]. Med Intensiva 2012; 36: 358-64. 